\section{Prospective decrease in progesterone concentrations in female lightweight rowers during the competition season compared with the off season: a controlled study examining weight loss and intensive exercise}

Fiona L Morris, Warren R Payne, John D Wark

\begin{abstract}
The purpose of this study was to monitor ovarian hormone function response to intense exercise and body weight changes in female athletes. Ovarian hormone function was evaluated in $\mathbf{1 2}$ female lightweight rowers and 10 age-height-weight matched sedentary controls. Ovarian hormone function was assessed during consecutive competition season and off season, by measurement of peak and average alternative day overnight urinary oestrone glucuronide $\left(E_{1} G\right)$ and pregnanediol glucuronide (PdG) excretion. Competition season was associated with a $5.8 \mathrm{~kg}(9.3 \%)$ body weight loss in the lightweight rowers. Significantly lower competition season peak and average urinary excretion of PdG were found in the lightweight rowers compared with the controls. Lower competition season peak and average urinary excretion of $E_{1} G$ were also found in the lightweight rowers compared with the controls, but the difference did not reach significance. The number of rowing training hours was a significant determinant of peak PdG excretion in the rowers $\left(R^{2}=\right.$ $0.40 ; p<0.02)$. The seasonal suppression of PdG excretion was associated with degree of weight loss $\left(R^{2}=0.46 ; p<0.01\right)$. The competition related decrease in $E_{1} G$ and PdG excretion for the lightweight rowers was predominantly restored during the off season when exercise intensity and duration were decreased and body weight increased. These results showed a significant $(p<0.05)$ reduction in progesterone metabolite excretion and a non-significant decrease in oestrone metabolite excretion associated with intensive competition season training loads and body weight reduction in female lightweight rowers. (Br F Sports Med 1999;33:417-422)
\end{abstract}

Keywords: oestrogen; progesterone; rowers; weight loss; training

Dr F Morris, Faculty of Communication, Health \& Science, School of Science, Edith Cowan University, 100 Joondalup Drive, Joondalup, Perth, Western Australia, Australia 6027.

Accepted for publication 2 September 1999 alterations in reproductive endocrinology in athletes, particularly during phases of intense
Exercise regimens among women have become increasingly strenuous, with research suggesting that increased training loads may be contributBiomedical and Sport competition season training. ${ }^{1-4}$ Training associated ovarian functional disturbances show a spectrum of reversible changes spanning primary and secondary amenorrhoea and oligomenorrhoea, ${ }^{5}$ anovulatory cycles, ${ }^{6}$ luteal phase deficiency, ${ }^{58}$ short luteal phase length, and delayed menarche. ${ }^{9} 10$ Few reports have documented the seasonal changes in reproductive endocrinology in athletes faced with seasonal increases in training intensity, to investigate whether intensive competition training contributes to altered ovarian function.

Lightweight rowing is a sport that, in addition to intensive competition season training, presents a second potential risk factor for altered ovarian hormone function, body weight reduction. Lightweight rowing requires the athletes to meet weight restrictions, usually below their normal body weight. Research suggests that low body weight and body weight loss may contribute to altered ovarian hormone function. ${ }^{11}{ }^{12}$ Athletes who lose body weight during training show more significant ovarian hormone function alterations than those who maintain body weight, despite the same training loads. ${ }^{1}$ The purpose of this study was therefore to monitor the ovarian hormones in female lightweight rowers during the competition season and off season training phases of a rowing season, to evaluate the relation between intensive training, weight reduction, and ovarian hormone function.

\section{Methods and procedures}

SUBJECTS

Twenty one lightweight rowers from the Australian national lightweight rowing programmes were voluntarily recruited for the study. A meeting was arranged for all interested rowers at the national training camp, where the study protocol was explained to each lightweight rower. An initial questionnaire was administered to the interested rowers, relating to their health, menstrual cycle history, number of menstrual cycles experienced during the year leading into the study, and oral contraceptive use. Exclusion criteria for the study were use of oral contraceptives, smoking, metabolism such as diabetes, renal disease, heart disease, or anaemia. Of the 21 lightweight rowers, nine were currently taking oral contraception and were excluded from commencing the study. The final cohort consisted of 12 or any disease that would interfere with bone 
female lightweight rowers. Ten sedentary control subjects matched for age, height, and off season body weight were voluntarily recruited for comparative purposes. Informed written consent was obtained from all subjects before testing, in accordance with protocols approved by the board of medical research and the ethics committee on research at the Royal Melbourne Hospital.

PHYSICAL ACTIVITY

Once during the competition season and once during the off season, the rowers completed a daily physical activity training diary, incorporating two days during a week and one weekend day. ${ }^{13}$ Details of physical activity participation included mode of training, total duration, distance, and intensity of activity. The information provided in the diaries was validated by checking against coaches' training records. The control subjects completed a similar physical activity participation diary, corresponding to the rowers' competition season.

MENARCHE, GYNAECOLOGICAL AGE, AND

MENSTRUAL HISTORY INDEX

Age of menarche was assessed by the recall method, requiring each subject to recall the approximate date of her first menstrual cycle. From this information, age of menarche in years and months was established. The recall accuracy of this method has been reported to be up to $80 \%$ if performed carefully. ${ }^{14}$ Gynaecological age was subsequently calculated as the number of years since the age of menarche.

INVESTIGATION OF URINARY OVARIAN FUNCTION Oestrogen and progesterone concentrations were estimated by monitoring the excretion of urinary oestrogen (oestrone glucuronide $\left(\mathrm{E}_{1} \mathrm{G}\right)$ ) and progesterone (pregnanediol glucuronide (PdG)) metabolites across a complete menstrual cycle during the competition season for both the rowers and control subjects and again in the off season for the lightweight rowers. Unfortunately the control subjects failed to provide a "rowing off season" hormone sample which is acknowledged as a limitation to this study. The competition season hormone samples were collected in April, before the national championships, with the off season hormone samples collected five months later in September. Commencing on day 1 of the cycle, urinary samples for measurement of $E_{1} G$ and PdG excretion were collected on every second day of one complete cycle during the competition season for the rowers and controls. Each subject was supplied with a collection kit containing 15 collection tubes, a calibrated urine collection jug, and a recording booklet. Subjects were instructed to record the sample collection time, which was the time from the last voiding at night to the collection of the first voided specimen the following morning. The urine sample was then diluted with distilled water, with the volume of distilled water being a proportion of the total collection time. A 100 $\mathrm{ml}$ urine sample was then collected into a tube, capped, dated, stored in the domestic refrigerator, and frozen at about $-20^{\circ} \mathrm{C}$. The samples were then delivered to the University of Melbourne for subsequent analysis.

Urinary $\mathrm{E}_{1} \mathrm{G}$ and PdG excretion was quantified using a three step homogeneous enzyme immunoassay as described previously and validated by comparison with established methods. ${ }^{15} 16$ The selected operating range for the $\mathrm{E}_{1} \mathrm{G}$ assay was $10-1000 \mathrm{nmol} / 24 \mathrm{~h}$ and for the PdG assays $0.1-30 \mu \mathrm{mol} / 24 \mathrm{~h}^{15}{ }^{17}$ The coefficient of variation for the $\mathrm{E}_{1} \mathrm{G}$ and $\mathrm{PdG}$ assays is about $10 \% .{ }^{15}{ }^{18}$ The interassay coefficients of variation for the high and low urinary $E_{1} G$ concentration were $5.2 \%$ and $4.9 \%$ respectively. The interassay coefficients of variation for the high and low urinary PdG concentration were $3.6 \%$ and $2.7 \%$ respectively. ${ }^{15}{ }^{16}$ All samples from a subject were measured in one assay run. All $\mathrm{E}_{1} \mathrm{G}$ and $\mathrm{PdG}$ values were expressed as $\mu \mathrm{mol} / 24 \mathrm{~h}$. Luteal phase was distinguished from the follicular phase by observing the initial rise in $\mathrm{E}_{1} \mathrm{G}$ levels, followed by a rapid rise in PdG above $9.0 \mu \mathrm{mol} / 24 \mathrm{~h}$ after ovulation. An integrated value for oestrogen and progesterone was calculated for each subject by multiplying the average daily $E_{1} G$ and PdG excretion level by the number of days of the menstrual cycle.

\section{LONGITUDINAL HORMONE MONITORING}

Longitudinal monitoring of $\mathrm{E}_{1} \mathrm{G}$ and $\mathrm{PdG}$ excretion across three menstrual cycles was carried out for one national level lightweight rower competing at the World Championships. She collected urine samples for ovarian hormone determination once during the domestic competition season in April, once before departing for the World Championships in June, and once during the off season in September.

\section{STATISTICAL ANALYSIS}

Statistical analysis was conducted using the SPSS software (version 8.0). Comparisons between rowers and controls were made for all physical characteristics, oestrogen and progesterone metabolite excretion rate, and ovulatory function by individual Student's $t$ tests. A nonparametric Kruskal-Wallis test was used to compare menstrual cycle phases and hormone concentrations in the controls and rowers across time (competition season/off season). Multiple regression analysis was performed to assess the relation between physical characteristics, body composition, physical activity levels, gynaecological history, and hormone excretion rates. Simple regression analysis was performed to assess the relation between changes in total body weight in the rowers and seasonal changes in oestrogen and progesterone metabolite excretion rate. The significance for all statistical analysis was set at $\mathrm{p}<0.05$.

\section{Results}

PHYSICAL CHARACTERISTICS

The 12 lightweight rowers and 10 control subjects were of similar age, height, and total body mass during the competition season. However, the lightweight rowers had a significantly lower percentage body fat content, a higher total lean 
Table 1 Baseline physical characteristics of the lightweight rowers and control subjects

\begin{tabular}{llll}
\hline Body composition & Rowers (n=12) & Controls (n=10) & p Value \\
\hline Age (years) & $21.5(3.5)$ & $22.0(3.5)$ & 0.73 \\
Height (cm) & $169.0(4.8)$ & $164.6(4.9)$ & 0.70 \\
Mass comp season (kg) & $56.5(2.5)$ & $57.5(4.2)$ & 0.75 \\
Mass off season (kg) & $62.0(3.1)$ & $57.5(4.2)$ & $0.05^{\star}$ \\
\% Fat comp season & $22.4(5.0)$ & $26.5(3.2)$ & $0.04^{\star}$ \\
Fat mass comp season (g) & $13.1(3.2)$ & $14.9(2.2)$ & 0.20 \\
Lean mass comp season (g) & $44.2(3.7)$ & $40.2(2.5)$ & $0.01^{\star}$ \\
Physical activity & & & \\
$\quad$ In season rowing (h) & $13.0(2.3)$ & $0.00(0.0)$ & $0.01^{\star}$ \\
In season rowing (km) & $163.0(22)$ & $0.0(0.0)$ & $0.01^{\star}$ \\
In season other sport (h) & $7.0(2.0)$ & $3.8(1.1)$ & $0.01^{\star}$ \\
\hline
\end{tabular}

Values are mean (SD)

*Significant difference between controls and rowers.

Table 2 Baseline ovarian function characteristics of the lightweight rowers and control subjects

\begin{tabular}{llll}
\hline & Rowers $(n=12)$ & Controls $(n=10)$ & $p$ Value \\
\hline Age at menarche (years) & $13.1(0.7)$ & $12.3(1.1)$ & $0.03^{\star}$ \\
Gynaecological age (years) & $8.4(3.7)$ & $9.7(3.4)$ & 0.18 \\
Menses previous year & $8.3(3.4)$ & $12.5(0.5)$ & $0.01^{\star}$ \\
\hline
\end{tabular}

Values are mean (SE).

* Significant difference between controls and rowers.

mass, and were heavier during the off season. The lightweight rowers also engaged in significantly more physical activity than the controls (table 1).

\section{OVARIAN FUNCTION}

Age at menarche for the lightweight rowers was 0.8 years later than for the controls (table 2). However, despite different menarcheal ages, the gynaecological ages in the two groups were similar. The lightweight rowers had had fewer menstrual cycles during the previous year and a lower menstrual history index, indicating fewer menstrual cycles experienced since menarche. During the competition season, the length of the rowers' menstrual cycle was significantly longer than the controls, a difference that was no longer evident during the off season. The average menstrual cycle length during the competition season was 47.9 (33.0) days for the lightweight rowers, decreasing to 28.3 (3.0) days during the off season. The off season menstrual cycle length for the lightweight rowers was similar to the menstrual cycle length of the control subjects at 27.0 (2.0) days (table 3). The significantly longer competition season menstrual cycle duration for the rowers was associated with a significantly longer follicular phase $(\mathrm{p}<0.01)$ and significantly shorter luteal phase $(\mathrm{p}<0.01)$.

Table 3 Seasonal menstrual cycle length and peak and average urinary excretion of oestrone glucuronide $\left(E_{1} G\right)$ and pregnanediol glucuronide (PdG) for the lightweight rowers during the competition season and off season and for the control subjects

\begin{tabular}{|c|c|c|c|c|}
\hline \multirow[b]{2}{*}{ Hormone } & \multicolumn{2}{|c|}{ Rowers $(n=12)$} & \multirow{2}{*}{$\begin{array}{l}\text { Controls } \\
(n=10)\end{array}$} & \multirow[b]{2}{*}{$p$ Valuc } \\
\hline & Competition & Off season & & \\
\hline Follicular phase (days) & $43.9(35.4)$ & $18.2(5.5)$ & $14.4(0.5)$ & $0.01 \star$ \\
\hline Luteal phase (days) & $4.1(5.9)$ & $10.2(5.2)$ & $12.6(1.8)$ & $0.01 \star$ \\
\hline Menstrual cycle length (days) & $47.9(33.0)$ & $28.3(3.0)$ & $27.0(2.0)$ & 0.01 * \\
\hline Peak $E_{1} G$ per cycle $(\mathrm{nmol})$ & $201(20)$ & $251(30)$ & $272(32)$ & 0.55 \\
\hline Average $E_{1} G$ per cycle $(\mathrm{nmol})$ & $110(14)$ & $152(14)$ & $143(11.3)$ & 0.31 \\
\hline Peak PdG per cycle $(\mu \mathrm{mol})$ & $6.4(3.4)$ & $11.6(1.3)$ & $13.0(0.8)$ & $0.01 \star$ \\
\hline Average PdG per cycle $(\mu \mathrm{mol})$ & $3.1(1.9)$ & $6.7(0.8)$ & $7.8(0.7)$ & $0.01 \star$ \\
\hline
\end{tabular}

Values are mean (SE).

* Significant difference between controls and rowers.

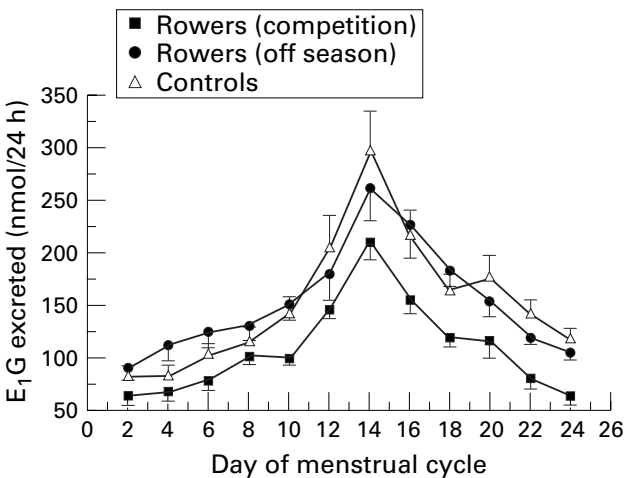

Figure 1 Seasonal urinary oestrone glucuronide $\left(E_{1} G\right)$ excretion during the menstrual cycle for the lightweight rowers during the competition season and off season and for the control subjects (mean (SEM)).

SEASONAL URINARY OVARIAN HORMONES

The rowers' peak and average urinary $\mathrm{E}_{1} \mathrm{G}$ and PdG excretion showed seasonal variations (table 3). Significantly lower peak and average progesterone metabolite excretion rates were recorded for the rowers during the competition season, differences that did not persist during the off season. The differences in the oestrogen metabolite excretion rates between the groups did not reach statistical significance (table 3).

\section{SEASONAL OESTROGEN PROFILES}

During the off season, the rowers and controls both showed the characteristic biphasic midcycle $\mathrm{E}_{1} \mathrm{G}$ peaks of 251 (30) and 272 (33) $\mathrm{nmol} / 24 \mathrm{~h}$ respectively, followed by a secondary $\mathrm{E}_{1} \mathrm{G}$ peak. During the competition season the rowers' urinary $\mathrm{E}_{1} \mathrm{G}$ excretion level was reduced to 201 (20) $\mathrm{nmol} / 24 \mathrm{~h}$. The seasonal difference in the rowers' $\mathrm{E}_{1} \mathrm{G}$ excretion level was not significant (fig 1).

SEASONAL PROGESTERONE PROFILES

Detailed monitoring of urinary PdG excretion indicated that the controls had a significantly higher peak PdG excretion at $13.0(0.8) \mu \mathrm{mol} / 24$ $\mathrm{h}$ compared with 6.4 (3.4) $\mu \mathrm{mol} / 24 \mathrm{~h}$ for the rowers during the competition season. Again no difference in peak PdG excretion existed between the groups during the off season. The controls also displayed higher average luteal phase $\mathrm{PdG}$ at $7.8(0.7) \mu \mathrm{mol} / 24 \mathrm{~h}$, when compared with the rowers during the competition season, at $3.1(0.9) \mu \mathrm{mol} / 24 \mathrm{~h}$. The differences in $\mathrm{PdG}$ excretion rates between groups were no longer evident during the off season (fig 2).

Multiple regression analysis indicated that the menstrual history index was a significant determinant of peak $\mathrm{E}_{1} \mathrm{G}$ excretion $\left(R^{2}=0.33\right.$; $\mathrm{p}<0.05)$ and average $\mathrm{PdG}$ excretion $\left(R^{2}=0.40\right.$; $\mathrm{p}<0.02)$ in the rowers. The total number of rowing hours was a significant determinant of peak PdG excretion $\left(R^{2}=0.33\right.$; $\left.<<0.05\right)$ in the rowers. No factors were significant predictors of average $E_{1} G$ excretion in the rowers. No factors were predictive of hormone excretion in the control subjects. 


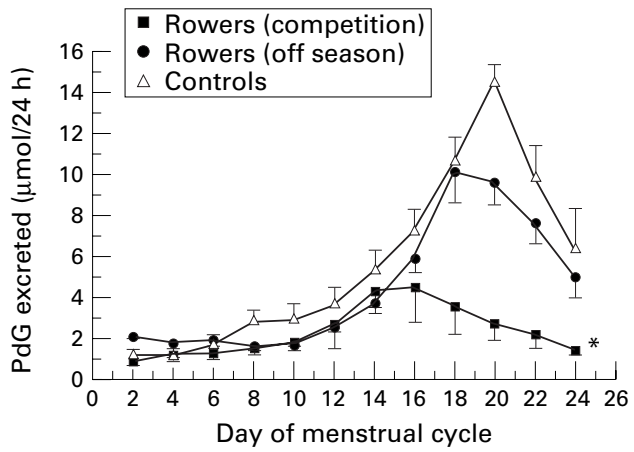

Figure 2 Seasonal urinary excretion of pregnanediol glucuronide ( $P d G)$ during the menstrual cycle for the lightweight rowers during the competition season and off season and for the control subjects. Values are mean (SEM). ${ }^{\star} p<0.05$ compared with values for lightweight rowers in the off season and the controls.
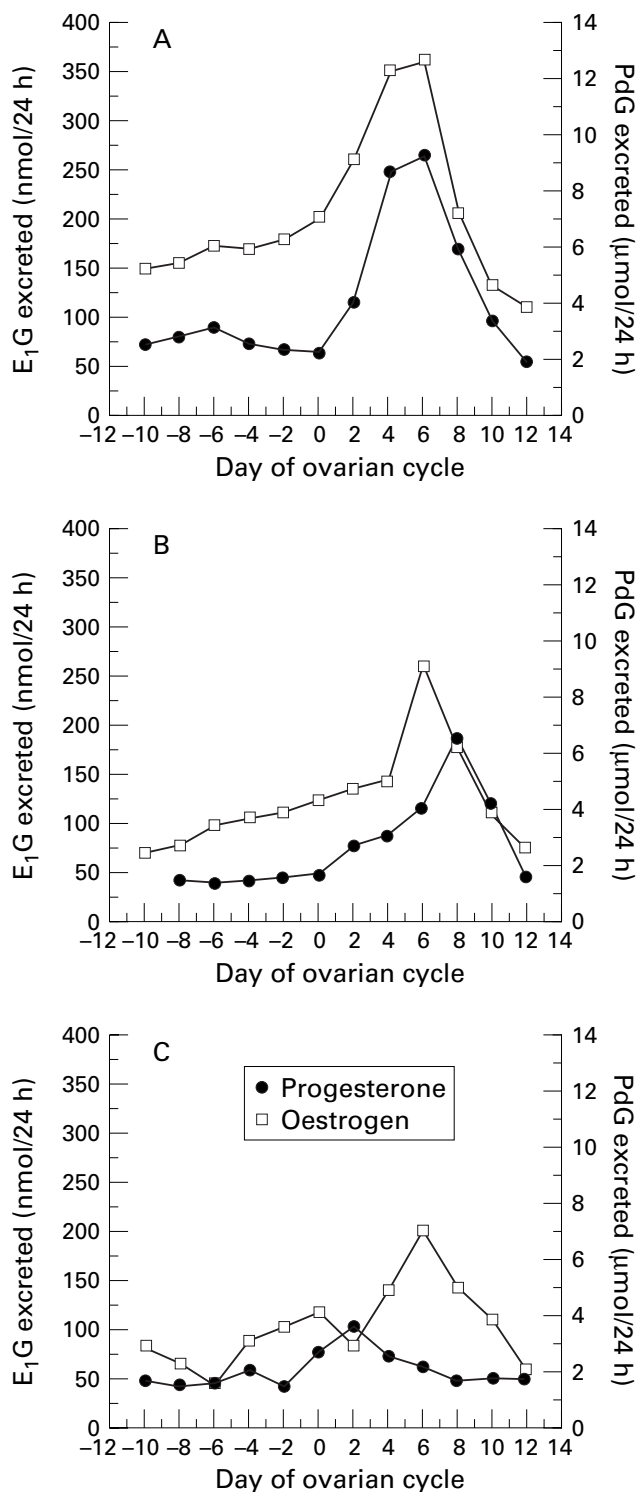

Figure 3 Longitudinal ovarian hormone excretion (oestrone glucuronide $\left(E_{1} G\right)$ and pregnanediol glucuronide $(P d G)$ ) for a lightweight rower leading up to the World Championships. The three collections were $(A)$ before the season in August (62.9 kg), (B) early competition season in April (58.5 kg), and (C) competition season before departing for the World Championships in fuly (57.9 kg). Body weights at the time of data collection are given in parentheses.
BODY WEIGHT AND OVARIAN HORMONE

EXCRETION

The lightweight rowers lost 5.8 (1.6) $\mathrm{kg}$ to reach competition weight, equivalent to $9.3 \%$ of their pre-season body weight. This seasonal weight loss was associated with significant reductions in peak and average progesterone metabolite excretion, with regression analysis indicating that the degree of weight loss was a significant determinant of the variance in progesterone metabolite excretion (adjusted $R^{2}=$ $0.46 ; \mathrm{p}<0.01)$. No significant differences were found between alterations in body mass and alterations in oestrogen metabolite excretion.

LONGITUDINAL HORMONE PROFILES IN THE LEAD UP TO THE WORLD CHAMPIONSHIPS

An in depth insight into ovarian hormone function and the response to increasingly strenuous training loads and body weight reduction was provided by examining the hormone profiles of one Australian lightweight rower in the lead up to the World Championships (fig 3). As the rower advanced towards the major rowing regatta, the reproductive hormone excretion was progressively reduced. Owing to the difficulties associated with travel, hormone collection was not possible for the final menstrual cycle.

\section{Discussion}

This study shows seasonal variation in ovarian hormone function in lightweight rowers associated with an intensive rowing training programme and competition season weight loss. Several factors confronting the lightweight rowers have been postulated as triggers for ovarian disturbance. These include exercise stress, stress associated with competition, body weight loss, altered body composition and body fat levels, and reduced energy intake. Owing to the multifaceted pathogenesis of menstrual disturbance, it is more than likely that the competition season alterations in ovarian hormone axis function observed in the lightweight rowers were a response to a combination of several of these factors.

The most noticeable change in ovarian hormone function for the lightweight rowers would have been the altered length of menstrual cycle duration, decreasing from 48 days during the competition season to 28 days during the off season. Competition season menstrual cycle was associated with a long follicular phase and a significantly shorter luteal phase. These documented changes in menstrual cycle phase duration are consistent with alterations reported in several female athletic populations engaged in increasingly intensive exercise training. ${ }^{58}$ However, it should be noted that alterations in menstrual cycle phases were not documented in all female lightweight rowers, indicating that the ovarian hormonal function response to increased training loads and weight loss is specific to each female athlete.

The lightweight rowers also had significantly lower progesterone metabolite excretion rates during the competition season, reductions that were reversed during the off season, resembling 
the hormone profiles of the control subjects. By monitoring seasonal ovarian function, this study shows a possible dynamic relation between exercise training loads and ovarian function. The high incidence of altered ovarian function induced by intensive exercise observed in this study parallels the incidence rates observed in other competitive female athletes ${ }^{17-20}$ and women exposed to increasingly strenuous workloads during the summer monsoons. ${ }^{21}$

The entire spectrum of altered ovarian hormone function was documented in one lightweight rower. In the lead up to the World Championships, her ovarian function was monitored across three menstrual cycles. The menstrual cycles two months before showed low oestrogen and progesterone metabolites, progressing to very low oestrogen and progesterone metabolites in the month preceding the championships, with no menstrual cycle reported while she was racing overseas. The dynamic relation between ovarian function and exercise was shown when ovarian hormone levels were restored during the off season when training intensity was reduced and body weight regained. The observation of the dynamic relation between exercise and altered ovarian function was consistent with research reporting ovarian disturbance in previously sedentary women who commenced training for a marathon. ${ }^{18}$ The luteal phase at baseline was classified as normal (12 days), but subsequently decreased in length as the intensity and length of the daily runs increased. The two cycles immediately before the marathon were anovulatory. ${ }^{18}$ The dynamic relation between luteal phase length, ovulation, and exercise was supported when the menstrual cycle phase returned to normal after the marathon when exercise training intensity decreased.

Another notable finding from this study was a link between body weight reduction and altered ovarian hormone function. The lightweight rowers in this study were confronted with enforced weight restrictions which had to be attained before the rower was eligible to compete. Weight loss for the lightweight rowers was $5.8 \mathrm{~kg}$, equivalent to $9.3 \%$ of their off season body weight. Low oestrogen and progesterone metabolites were also more prevalent in the lightweight rowers who had to lose the greatest amount of body weight to reach race weight. This body weight loss was associated with the suppression of progesterone metabolite concentrations during the competition season. As previous research has shown, abrupt and pronounced weight loss, coupled with the physical stress of exercise, is often associated with ovarian disturbance. ${ }^{21}$ Seasonal suppression of fertility was documented with both large weight losses of $5-10 \%$ of body mass ${ }^{22}$ and body weight loss as small as $2 \%$ of body mass $(0.9 \mathrm{~kg}),{ }^{20}$ suggesting that even a relatively small change in body weight of $1-2 \mathrm{~kg}$ around the critical level can influence ovarian function. ${ }^{23}$

This study indicates that strenuous physical exercise, compounded by weight loss, can alter the ovarian hormone function in some female athletes. Although the association of athletic training with reproductive disturbance is well established, we show here that greater training loads and weight loss are associated with an increased prevalence of altered ovarian hormone function. Many of the documented ovarian disturbances remained indistinguishable from ovulatory cycles because of the preservation of regular menstrual cycle length and would be regarded as perfectly normal by the lightweight rowers. Lightweight rowers with the highest pre-season weight, who needed to lose the most weight to be eligible to race in the lightweight rowing category, had the lowest body fat levels, trained the longest, and had had a history of menstrual cycle irregularity were most likely to incur altered ovarian hormone function. These findings are important when selecting athletes suitable to compete in the lightweight rowing category and maintain ovarian hormone function.

Contributors: F M initiated and coordinated the formulation of the project design and hypothesis, conducted the data collection, performed the data analysis, contributed to the discussion and conclusion section, and contributed to the writing of the paper. W P was involved in the formulation of the project design and hypothesis, data analysis, and contributed to project design and hypothesis, data analysis, and contributed to
the discussion and conclusion sections. J W was involved in the the discussion and conclusion sections. J W was involved in the and contributed to the discussion and conclusion sections.

1 Bullen BA, Skrinar GS, Beitins IZ, et al. Induction of menstrual disorders by strenuous exercise in untrained women. strual disorders by strenuous exer.
$N$ Engl $\mathcal{F}$ Med 1985;312:1349-53.

2 Dale D, Saris W, TenHoor F. Does exercise have an additional effect on weight loss and resting metabolic rate? Int $\mathcal{F}$ Obes 1979;11:867-75.

3 Feitch CB, Johnson TS, Martin BJ, et al. Secondary amenorrhea in athletes. Lancet 1978;2:1145.

4 Pakainen HR, Pararinen AJ, Kirkinen P, et al. Physical exercise-iduced changes and season-associated differences in the pituitary-ovarian function of runners and joggers. $\mathcal{F}$ Clin Endocrinol Metab 1985;60:416-22.

5 Loucks A. Effects of exercise training on the menstrual cycle: existence and mechanisms. Med Sci Sports Exerc 1990;22:275-80

6 Prior J, Vigna Y, Schechter M, et al. Spinal bone loss and ovulatory disturbances. $N$ Engl F Med 1990;323:1221-7.

7 Beitins IZ, McCarther JW, Turnbull BA, et al. Exercise induces two types of human luteal dysfunction: confirmainduces two types of human luteal dysfunction: confirma-
tion by urinary free progesterone. F Clin Endocrinol Metab tion by urinary free

8 Bonen A, Belcastro AN, Ling WY, et al. Profiles of selected hormones during menstrual cycles of teenage athletes. $\mathcal{F}$ Appl Physiol 1981;50:545-51.

9 Malina RM. Menarche in athletes: a synthesis and hypothesis. Ann Hum Biol 1983;10:1.

10 Warren MP. The effects of exercise on pubertal progression and reproductive function in girls. F Clin Endocrinol Metab 1980;51:1150-7.

11 Pirke MC, Schweiger U. Dieting influences the menstrual cycle: vegetarian verses nonvegetarian diet. Fertil Steril 1986;46:1085

12 Pirke KM, Schweiger U, Strwitzki T. Dieting causes menstrual irregularities in normal weight women through impairment of luteinizing hormone. Fertil Steril 1989;51: 263-8.

13 Bouchard C, Tremblay A, Leblanc C. A method to assess energy expenditure in children and adults. Am $\mathcal{F}$ Clin Nutr 1983;37:461-7.

14 Ross WD, Marfell-Jones MJ. Kinathropometry. In: MacDougal JD, Wenger HA, Green HJ, eds. Physiological testing of the high performance athlete. Champaign, IL: Human Kinetics, 1991:224-305.

15 Brown JB, Blackwell LF, Cox RI, et al. Chemical and homogeneous enzyme immunoassay methods for the measurement of estrogens and pregnanediol and the gluconides in urine. In: Non-radiometric assays: technology and application in polypeptide and steroid hormone detection. New york: Alan R Liss Inc, 1988:119-38.

16 Bonen A, Belcastro AN, Ling WY, et al. Profiles of selected hormones during menstrual cycles of teenage athletes. $\mathcal{F}$ Appl Physiol 1981;50:545-51.

17 Broocks A, Pirke K, Schweiger U, et al. Cyclic ovarian function in recreational athletes. F Appl Physiol 1990;68:200386.

18 Prior JC, Ho Yeun B, Clement P, et al. Reversible luteal phase changes and infertility associated with marathon phase changes and infertility
training. Lancet 1982;1:269-70.

19 Rogol A, Weltman J, Seip R, et al. Gonadotropin secretion in female runners. In: Laron A, Rogol A, eds. Hormones and sport. New York: Raven Press, 1989:114-66. 
20 Panter-Brick C. Seasonality of reproductive function and weight loss in rural Nepali women. Human Reprod 1993;8: weight loss

21 Ellison PT, Peacock NR, Lager C. Ecology and ovarian function among Lese women of the Ituri forest, Zaire. $A m$ f Phys Anthropol 1989;78:519-26.
22 Bates GW, Bates SR, Whitworth NS. Reproductive failure in women who practice weight control. Fertil Steril 1982;37: women

23 Frisch

Frisch R, Gotz-Welbergen A, McArthur J. Delayed menarche and amenorrhea of college athletes in relation to age of onset of training. ҰAMA 1981;246:1559-63.

\section{Take home message}

Female athletes undertaking strenuous training loads need to be aware that intensive training is a potential trigger for altered ovarian hormone function, and that the greater the training loads, the greater the risk. In addition, strenuous training accompanied by weight loss will compound the potential risk of altered ovarian hormone function. Alterations in ovarian hormone function are very individual and appear to be a temporary adaptation, returning to normal once the training loads are reduced and weight regained. 